\title{
INEQUALITIES FOR THE ZEROS OF THE ASSOCIATED ULTRASPHERICAL POLYNOMIALS
}

\author{
P. D. SIAFARIKAS
}

Abstract. Using a functional analytic method based on the three-term recurrence relation of orthogonal polynomials, we study the monotonicity of the zeros of the associated ultraspherical (or Gegenbauer) polynomials, and we give some inequalities for the largest zeros.

Mathematics subject classification (1991): 33C45.

Key words and phrases: Inequalities, associated ultraspherical polynomials.

\section{REFERENCES}

[1] R. Askey And J. Wimp, Associated Laguerre and Hermite polynomials, Proc. Roy. Soc. Edinburgh 96 (1984), 15-37.

[2] P. BaRRUCAND And D. Dickinson, On the associated Legendre Polynomials, Orthogonal Expansions and Their Continuous Analogues, Southern Illinois University, Carbandale, 1967, pp. 43-50.

[3] J. Bustoz And M. E. Ismail, The associated Ultraspherical Polynomials and their $q$ - Analogues, Canad. I. Math 34 (1982), 718-736.

[4] D. K. Dimitrov, On a conjecture concerning monotonicity of zeros of Ultraspherical polynomials, J. Approx. theory 85(1) (1996), 88-97.

[5] A. ELBERT AND A. LAFORGIA, On the zeros of associated polynomials of clasical orthogonal polynomials, Differential and Integral Equations 6 (1993), 1137-1143.

[6] A. Elbert AND P. D. SiafariKas, Monotonicity properties of the zeros of Ultraspherical Polynomlals, J. Appr. Theory (to appear).

[7] C. C. GRosjean, Theory of recursive generation of systems of orthogonal polynomials: An Illustrative example, J. Comput. Appl. Math. 12, 13 (1985), 299-318.

[8] C. C. GrosjeAn, The weight functions, generating functions and miscellaneous properties of the sequences of orthogonal polynomials of the second kind associated with Jacobi and the Gegenbauer polyomials, J. Comput. Appl. Math. 16(3) (1986), 299-318.

[9] W. HAHN, Under Orthoganadpolyome mit drei parameteren, Deutsche Math. 5 (1940-41), 373-378.

[10] E. K. IFANTIS, A Theorem concerning differentiability of eigenvectors and eigenvalues with some applications, Appl. Anal. 28 (1988), 257-283.

[11] E. K. Ifantis, Concavity and Convexity of eigenvalues, Appl. Anal. 41 (1991), 209-220.

[12] E. K. Ifantis, Perron-Frobenius-type theorems for tridiagonal operators, Appl. Anal. 44 (1992), 159-169.

[13] E. K. Ifantis AND P. N. PANagopoulos, On the zeros of a class of polynomials defined by a three term recurrence relation, J. Math. Anal. Appl. 182 (2) (1994), 361-370..

[14] E. K. IFANTIS AND P. D. SIAFARIKAS, Differential inequalities on the greatest zero of Laguerre and Ultraspherical polynomials, Actas del VI Simposium on Palinomios Orthoganales y Aplicationes, Gijon, Spain, 1989, pp. 187-197.

[15] E. K. IFANTIS AND P. D. SIAFARIKAS, On the zeros of a class of polynomials including the generalized Bessel polynomials, in: J. S. Dehesa Ed., Orthogonal polynomials and their Applications, J. Comput. Appl. Math. 49 (1993), 103-109. 
[16] E. K. Ifantis AND P. D. SiAfariKas, Differential Inequalities and Monotonicity properties of the zeros of Associated Laguerre and Hermite polynomials, International joint Symposium on Special functions and Artificial Intelligence, Torino Italy, 1993, in Annals of Numerical Mathem. 2 (1995), 79-91.

[17] E. K. IFANTIS AND P. D. SIAFARIKAS, Pertrurbation of the coefficients in the recurrence relation of a class of polynomials, 4th International Symposium on Orthogonal polynomials and their Applications, Evian-France, 1992, in J. Comput. Appl. Math. 57 (1995), 163-170.

[18] E. K. IFANTIS AND P. D. SiAfARIKAS, An alternative proof of a theorem of Stieltjes and related results, J. Comput. Appl. Math. 65 (1995), 165-172.

[19] E. K. Ifantis, C. G. Kokologiannaki and P. D. Siafarikas, Newton Sum rules and monotonicity properties of the zeros of scaled co-recursive Associated Polynomials, Math. Anal. Appl. 3(4) (1996), 486-497.

[20] M. E. ISMAIL AND X. LI, Bounds on the extreme zeros of orthogonal polynomials, Proc. Amer. Math. Soc. 115 (1992), 131-140.

[21] M. E. IsmaIL, Monotonicity of zeros of orthogonal polynomials, q-Series and Partitions (D Stanton, ed.), Springer-Verlag, New York, 1989, pp. 177-190.

[22] M. E. H. ISMAIL, The variation of zeros of certain orthogonal polynomials, Adv. Appl. Math, 8, 1987, pp. 111-119.

[23] M. E. A. Ismail AND R. ZhANG, On the Hellmann-Feynman theorems and the variation of zeros of certain special functions, Adv. Appl. Math 9 (1988), 439-446.

[24] M. E. H. IsmaIl AND M. E. MuldOON, A discrete approach to monotonicity of zeros of orthogonal polynomials, Trans. Amer. Math. Soc. 323(1) (1991), 65-78.

[25] A. LAFORGIA, Monotonicity properties for the zeros of orthogonal polynomials and Bessel functions, Edited by C. Brezinski, A. Draux, A. Magnus, P. Maroni and A. Ronveaux, Lecture Notes in Mathematics Springer-Verlag, New York (1985), 267-277.

[26] S. LeWANOWICZ, Results on the associated Jacobi and Gegenbauer polynomials, in: J. S. Dehesa Ed., Orthogonal polynomials and their Applications, J. Comput. Appl. Math. 49 (1993), 137-143.

[27] J. T. LEWIS AND M. E. MULDOON, Monotonicity and convexity properties of zeros of Bessel functions, SIAM J. Math. Anal. 8 (1977), 171-178.

[28] A. Ronveaux, Fourth order differential equations for numerator polynomials, J. Phys. A 21 (1988), $749-753$.

[29] A. RonVEAUX, Fourth order differential equations and orthogonal polynomials of the Laguerre-Hahn class, in: C. Brezinski, L. Gori and A. Ronveaux, Eds., Orthogonal Polynomials and their Applications, IMACS Ann. Comput. Appl. Math. 9 (Baltzer, basel, 1991), 379-385.

[30] J. WIMP, Explicit formulas for the asociated Jacobi polynomials and some applications, Canad. J. Math. 39 (1987), 983-1000.

[31] A. ZARZO, A. RONVEAUX AND E. GODOY, Fourth order differential equation satisfied by the associated of any order of all classical orthogonal polynomials. A study of their distribution of zeros, In: I. S. Dehesa Ed., Orthogonal Polynomials and their Applications, in J. Comput. Appl. Math 49 (1993), 349-359. 